\title{
Tuning the Resonance Frequency of Surface Plasmons Localized in Au-Ag Bimetallic Hollow Nanorods In-situ in a Transmission Electron Microscope
}

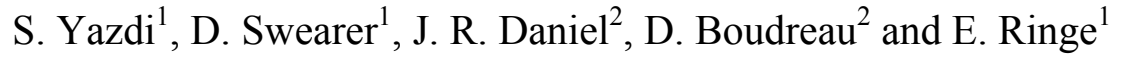 \\ ${ }^{1 .}$ Department of Materials Science \& Nano Engineering (MSNE), Rice University, Houston, Texas \\ United States \\ 2. The Center for Optics, Photonics and Lasers (COPL), Department of Physics, Laval University, \\ Québec, Canada
}

Metallic nanostructures can couple with electromagnetic radiation exciting collective oscillations of conduction electrons in resonance with the incident radiation. By tailoring the shape, size, composition, surrounding environment and interior structure of metallic nanostructures, it is possible to tune the resonance frequency of such localized surface plasmon resonances (LSPRs). Taking the advantage of these unique optical properties in metallic nanostructures, researchers have demonstrated a wide range of novel applications from photocatalysis, nanophotonics, and solar cells to biomedicine [1]. This application range can be extended significantly further if the frequency of LSPR can be tuned reversibly in individual metallic particles using external stimuli such as electric field, electric current, light or magnetic field. Here, we report on shifting reversibly the frequency of LSPR in individual Au-Ag hollow nanorods by using an electron beam in-situ in a transmission electron microscope (TEM).

The hollow bimetallic Au-Ag nanorods used in this study were synthesised by galvanic replacement of $\mathrm{Ag}$ templates with $\mathrm{Au}$ in an Au-salt solution [2]. Cross sectioning the nanorods using focused ion beam (FIB) confirms the hollow structure of nanorods (see Figure 1). By using an electron beam in a TEM we moved the material within the interior of the hollow nanorods and precisely positioned the hollow region in individual nanorods. This process is controllable and reversible, meaning that the hollow region can be placed at different positions within the nanorods and brought back to its initial position using the electron beam without changing the nanorod shape. Moving the hollow region within the nanorods shifts the frequency of the LSPR, and we used monochromated scanning transmission electron microscopy and electron energy loss spectroscopy (STEM-EELS) to measure this shift with high spatial resolution (see Figure 2). In-situ moving the hollow regions and monochromated STEM-EELS measurements were carried out at $80 \mathrm{kV}$ in a double Cs corrected FEI Titan Themis ${ }^{3}$ 60-300 S/TEM.

\section{References:}

[1] K. A. Willets et al. Annu. Rev. Phys. Chem. 58 (2007): 267-297.

[2] X. Xia et al. Advanced Materials 25.44 (2013): 6313-6333. 
Figure 1. FIB-prepared cross-section showing the hollow structure of Au-Ag nanoparticles. A $30 \mathrm{kV} \mathrm{Ga}$ ion beam in an FEI Helios 660 dual beam microscope was used to section the nanoparticles after protecting them from FIB damage with an electron beam deposited $\mathrm{Pt}$ layer. The SEM image is acquired at $52^{\circ}$ tilt.

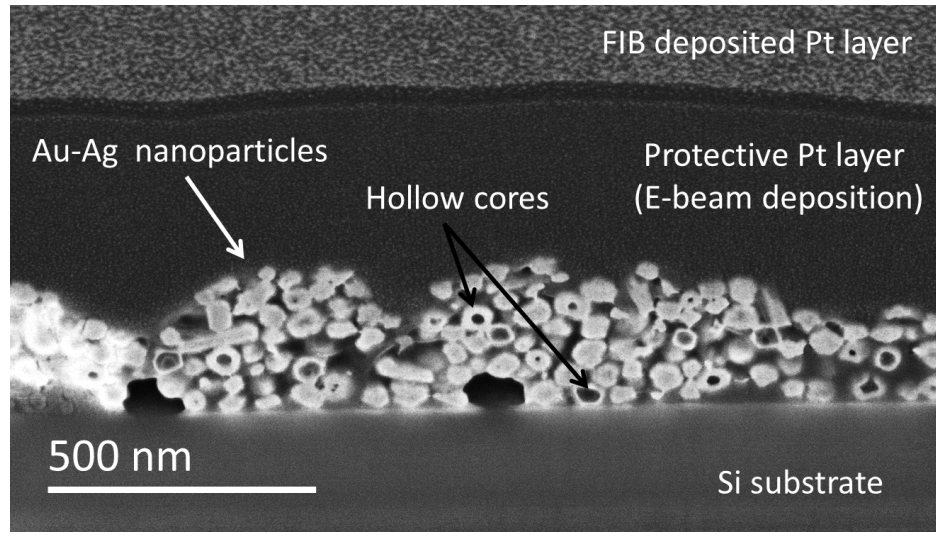

a)

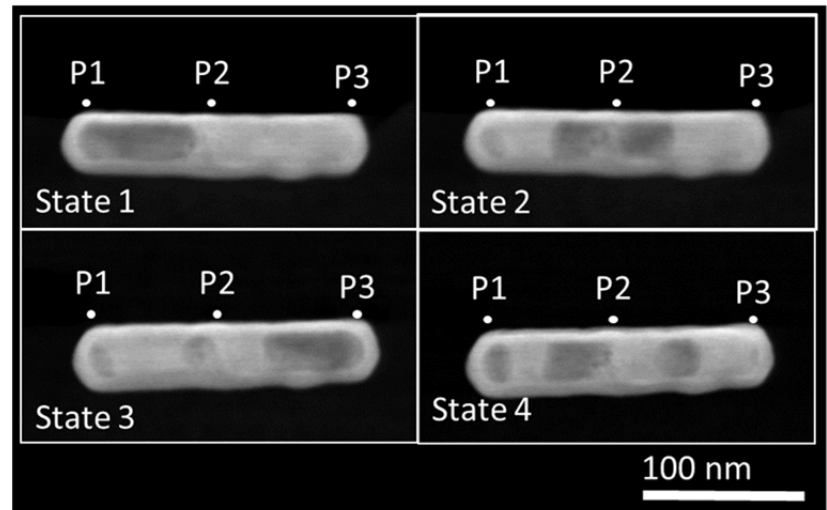

c)

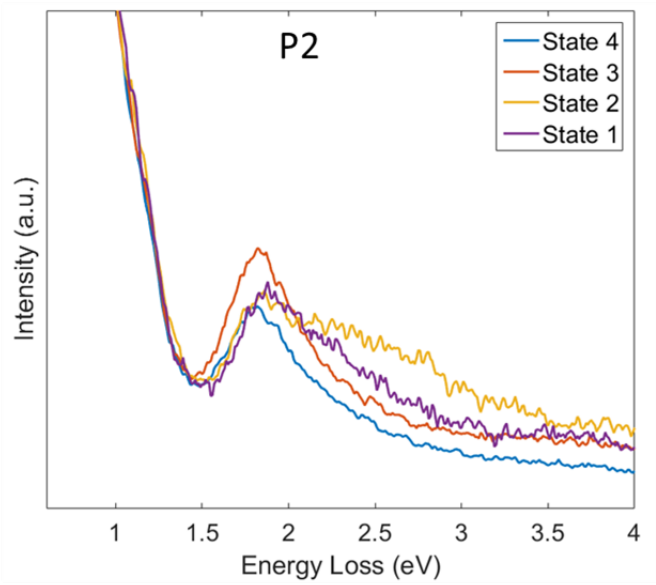

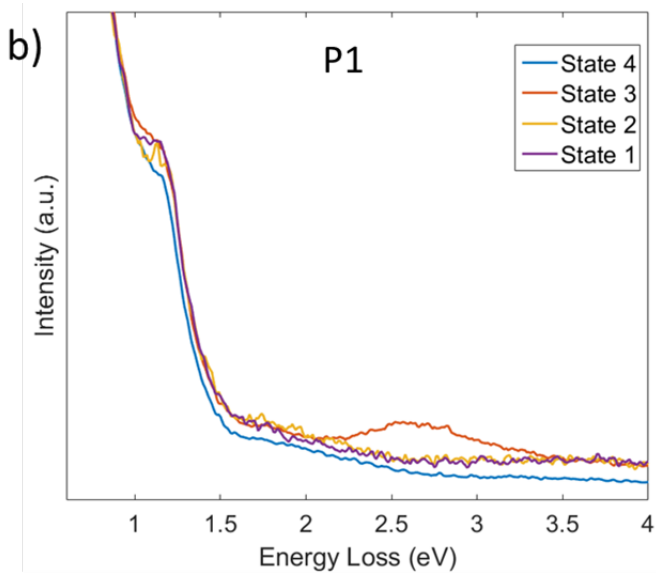

d)

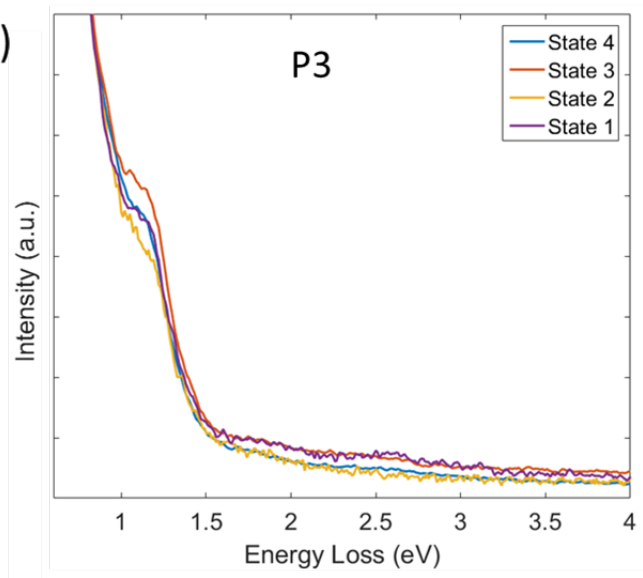

Figure 2. a) HAADF-STEM images of an individual hollow Au-Ag nanorod for four different positions of the hollow region (state 1, 2, 3 and 4), controlled by the electron beam. b, c and d) Raw monochromated EEL spectra acquired at three different positions (marked with P1, P2 and P3 on the HAADF-STEM images in a) at different states. 